\title{
Determinação de Setores de Atendimento em uma Concessionária de Energia
}

C.E.S. COSTA ${ }^{1}$, Programa de Pós-Graduação em Métodos Numéricos em Engenharia, Universidade Federal do Paraná (UFPR), Cx.P. 19011, 81531-980, Curitiba, PR, Brasil

D.M.B. COSTA2, Departamento de Desenho e Programa de Pós-Graduação em Métodos Numéricos em Engenharia, Universidade Federal do Paraná (UFPR), Cx.P. 19081, 81531-990, Curitiba, PR, Brasil

A.R.T. GÓES ${ }^{3}$ Programa de Pós-Graduação em Métodos Numéricos em Engenharia (UFPR) e Escola Municipal Planalto dos Pinheiros (EMPP), R. Manoel Ribas, n. 3561 - Jardim Planalto, 83708-350, Araucária, PR, Brasil.

Resumo. Este trabalho propõe a utilização de técnicas de Pesquisa Operacional para determinar agrupamentos de clientes (setores de atendimento) de uma concessionária de energia elétrica, com o objetivo de diminuir o tempo de execução dos serviços solicitados pelos clientes, através de uma melhor distribuição de tarefas entre as equipes disponíveis. O trabalho foi aplicado em uma das Agências da COPEL em Curitiba. Para obtenção dos resultados foram utilizados o algoritmo das p-medianas capacitado e algoritmos genéticos. Os vários testes realizados mostraram que os modelos matemáticos aplicados resolvem melhor o problema do que o método manual atualmente utilizado pela empresa.

\section{Introdução}

Em Curitiba, a Companhia Paranaense de Energia (COPEL) é dividida em cinco grandes áreas de atendimento denominadas Agências. Cada Agência é dividida em várias Rotas de Leitura. A figura 1 mostra a divisão da Agência do Portão, objeto deste trabalho. Para facilitar a distribuição de tarefas entre as diversas equipes que fazem o atendimento às solicitações dos clientes da empresa, várias Rotas de Leitura adjacentes são agrupadas formando Setores de Atendimento. Atualmente, a determinação destes Setores é feita manualmente, o que acarreta em uma divisão não homogênea de tarefas por equipe. Este trabalho pretende mostrar que pequenas modificações na situação atual podem acarretar em grandes melhorias no atendimento ao cliente.

\footnotetext{
${ }^{1}$ clarices@copel.com

${ }^{2}$ deise@ufpr.br

3 andersonroges@click21.com.br
} 


\section{Avaliação da Situação Atual}

A principal função das Agências da COPEL é atender às solicitações de seus clientes, consumidores de energia elétrica. Para tanto, é necessário designar as equipes disponíveis para os Setores de Atendimento, de maneira a executar os serviços solicitados dentro dos prazos estipulados à Companhia.

A Agência analisada possui 48 Rotas de Leitura, que são atendidas por 6 equipes de eletricistas. A designação dos serviços para as seis equipes é feita manualmente, utilizando-se o mapa da figura 1.

Ao solicitar um serviço à COPEL, o cliente informa o número da Rota de Leitura constante na sua fatura de energia elétrica, o que determina à qual Setor de Atendimento sua solicitação será encaminhada. Atualmente há sete setores e somente seis equipes, assim, uma equipe atende os setores 1 e 2 e as demais atendem cada uma um Setor.

Como os diversos serviços têm tempos médios de execução diferentes, o número de solicitações recebidas foi transformado em minutos necessários para atender às solicitações. O quadro 1 mostra que a distribuição atual de tarefas é ineficiente, pois o Setor 1 atende 5343 minutos à mais que o Setor 5 , o que é equivalente à 89,05 horas, ou 11,13 dias (de 8 horas) trabalhados.

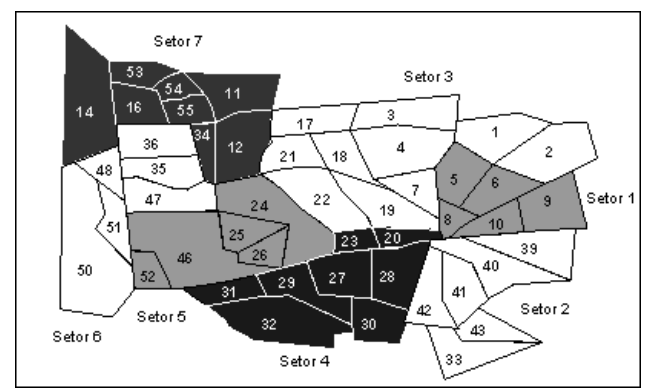

Figura 1: Divisão atual da Agência em sete Setores de Atendimento.

\begin{tabular}{|c|c|c|}
\hline Equipe & Setor de Atendimento & Tempo Necessário (minutos) \\
\hline 1 & $1 \mathrm{e} 2$ & 9894 \\
\hline 2 & 3 & 9041 \\
\hline 3 & 4 & 8233 \\
\hline 4 & 5 & 5527 \\
\hline 5 & 6 & 4551 \\
\hline 6 & 7 & 6127 \\
\hline
\end{tabular}

Quadro 1: Minutos despendidos, por equipe, para execução de tarefas, no mês de dezembro/04.

\section{Metodologias Utilizadas}

Para determinar regiões de atendimento, utilizou-se o modelo das p-medianas capacitado (método exato) e um Algoritmo Genético desenvolvido especialmente para o 
problema de formação de regiões (método heurístico).

\subsection{O Problema das $P$-Medianas}

O uso do modelo das $p$-medianas pode ser visto como o problema de tomar os $N$ vértices de um grafo e agrupá-los em $p$ conjuntos, em torno de um vértice mediano, onde existe ou deveria existir uma facilidade. Esse processo produz $p$ regiões (Setores) cujas respectivas medianas são as localizações das facilidades e cada um dos demais vértices do grafo pertence a uma das regiões cuja mediana seja a mais próxima. [9].

\subsection{Algoritmo Genético (AG)}

AG é um procedimento iterativo que mantém uma população de indivíduos, que representam as possíveis soluções para um determinado problema. [6].

A meta heurística utilizada neste trabalho, inspirada em [4], utiliza indivíduos com vários cromossomos como possível solução do problema e tem a característica de que a configuração de um cromossomo depende da configuração de todos os outros que compõem o mesmo indivíduo.

\section{Aplicação da Metodologia ao Problema Real}

Na resolução do problema real devemos determinar o melhor agrupamento de Rotas de Leitura em Setores de Atendimento, de maneira a homogeneizar a distribuição dos serviços entre as diversas equipes de eletricistas, levando em consideração a distância percorrida entre as Rotas de Leitura e os minutos trabalhados em cada Setor.

\subsection{Abordagem do Problema Real Através de Método Exato}

Nesta abordagem o problema é modelado matematicamente como um problema de p-medianas. A seguir estão detalhadas as etapas realizadas.

Para construção do grafo $G=(N, A)$, com $N=1,2, \ldots, 48$ vértices, cada Rota de Leitura da figura 1 foi substituída por um vértice, geograficamente próximo ao seu geocentro. Este vértice representa o ponto que concentra todas as solicitações de serviços daquela Rota. A distância entre os vértices é dada pela distância entre os geocentros das Rotas. A cada vértice $i \in N$ associou-se o peso $m_{i}$, que representa os minutos trabalhados na rota $(i)$, e a cada par de vértices $(i, j) \in A$ está associada uma distância $d(i, j)$. A matriz $\left[d_{i j}\right]$ reúne as distâncias entre todos os pares de vértices.

Como cada serviço demanda uma quantidade de tempo diferente para ser executado, observou-se que o número de solicitações recebidas por mês não forneceria uma análise real da situação. Assim sendo, a quantidade de serviços solicitados foi transformada em quantidade de minutos necessários para executar as solicitações, de acordo com as médias de tempo de execução de cada tarefa, obtidas pela observação empírica das equipes. 
As capacidades $M(k)$ de cada mediana $(k=$ dez, jan, ... mai $)$, foram obtidas da seguinte forma: $M(k)=T(k) / p$. Onde $T(k)$ é a demanda total de solicitações (em minutos) para o mês $k$ e $p$ representa o número de Setores de Atendimento desejados.

Como a obtenção da igualdade dos minutos trabalhados por equipe é improvável na maior parte das situações, incluíram-se no modelo os percentuais de tolerância $\alpha$ e $\beta$ multiplicados por $M(k)$. Assim, a melhor distribuição de tarefas é obtida com menores valores de $\alpha$ e $\beta$.

A formulação do problema das p-medianas capacitado, baseado em [8], é como segue

$$
\begin{gathered}
\operatorname{Min} \sum_{i=1}^{n} \sum_{j=1}^{n} d_{i j} x_{i j} \\
\sum_{i=1}^{n} x_{i j}=1 \quad(j=1,2, \ldots, n) \\
\sum_{i=1}^{n} x_{i i}=p \\
(1-\alpha) \cdot M x_{i i} \leq \sum_{j=1}^{n} m_{j} x_{i j} \quad(i=1,2, \ldots, n) \\
\sum_{j=1}^{n} m_{j} x_{i j} \leq(1-\beta) \cdot M x_{i i} \quad(i=1,2, \ldots, n) \\
x_{i j}=0 \text { ou } 1
\end{gathered}
$$

onde $D_{i j}=\left[d_{i j}\right]$ é a matriz das distâncias, $m_{j}$ é a demanda de solicitações da Rota de Leitura $j$, e as restrições em: (4.2) garantem que todo vértice $x_{j}$ é alocado a um e somente um vértice mediana $x_{i} ;(4.3)$ garantem que existem apenas $p$ vértices medianos; (4.4) garantem que as alocações só podem ser feitas aos vértices medianos; (4.5) garantem que a capacidade das medianas seja limitada inferiormente pela tolerância $\alpha$; (4.6) garantem que a capacidade das medianas seja limitada superiormente pela tolerância $\beta ;(4.7)$ definem a variável $x_{i j}$ como binária.

Para aumentar o espaço de busca por medianas, aumentou-se o número de vértices do grafo através da inserção manual de centróides fictícios no mapa, com pesos (minutos) igual a zero. A criação dos centróides fictícios deu-se inserindo uma malha no mapa utilizado e tomando-se as coordenadas de alguns novos pontos eqüidistantes, independentes dos centróides reais.

As simulações foram feitas considerando somente os 48 vértices existentes (abordagem 1) e considerando os 48 vértices existentes mais 26 fictícios (abordagem 2). 


\subsection{Abordagem do Problema Real Através de Algoritmo Genético}

Para este trabalho, um indivíduo factível é formado por tantos cromossomos quanto o número de Setores desejado. Em cada cromossomo $(j)(j=1,2, \ldots$, número de Setores desejado) tem-se

alelo $(i)= \begin{cases}1 & \text { se a rota leitura }(i) \text { é atendida pela equipe }(j) i=(1,2, \ldots, 48) ; \\ 0 & \text { caso contrário. }\end{cases}$

A heurística desenvolvida para geração da população inicial exige certas características na população: adjacência entre Rotas atendidas por uma mesma equipe, uma Rota deve ser atendida por somente uma equipe e a quantidade de minutos atendidos por equipe deve estar dentro do fator de tolerância definido pelo usuário. Nos indivíduos com melhores valores de função de aptidão (menor distância entre todas as Rotas de Leitura atendidas por um determinado Setor de Atendimento), aplicaram-se os operadores genéticos de crossover e mutação. O algoritmo é encerrado quando o novo indivíduo apresentar o total de minutos trabalhados por Setor dentro da tolerância definida nos dados de entrada ou se um número predeterminado de iterações é alcançado.

\section{Resultados Computacionais}

Para resolver o problema das $p$-medianas capacitado (método exato), foi desenvolvido um programa computacional em Visual Basic (VB) para geração da Função Objetivo do problema e suas restrições. Os dados de entrada, informados pelo usuário, são: o número de Setores desejado, o Fator de Tolerância aceito e o mês a ser simulado. O programa gera um arquivo de dados que é utilizado pelo software LINGO 6.0 para resolução do problema. Com 48 centróides são 2304 variáveis binárias e 2450 restrições, e com 74 centróides são 5476 variáveis e 5700 restrições.

As simulações foram realizadas nas dependências da Universidade Federal do Paraná (UFPR), pois a empresa não possui licença para utilização do software LINGO 6.0.

\subsection{Simulações do Método Exato}

As buscas pela solução ótima do problema foram iniciadas utilizando-se um fator de tolerância de $10 \%$ porém, quando a mesma não era alcançada, tal fator era aumentado gradativamente até o limite de $25 \%$. Em apenas $10 \%$ das simulações a resposta ótima do modelo não foi obtida com valores iguais ou inferiores à $25 \%$.

A figura 2, mostra a melhor sugestão de agrupamentos obtida para o mês de dezembro de 2004, dada pela abordagem 1, que apresentou a melhor distribuição de "minutos" por equipe, diminuindo a diferença entre as equipes com maior e menor demandas de solicitações. O quadro 2 mostra que, utilizando-se a divisão de Setores sugerida pela abordagem 1 do algoritmo das $p$-medianas, a diferença entre os Setores com maior e menor demanda de solicitações (Setores 4 e 1, respectivamente) cairia 
para 1.215 minutos, o que é equivalente à 20,25 horas. Transformadas em dias (de 8 horas) trabalhados, a diferença é de apenas 2,53 dias.

\begin{tabular}{|c|c|c|}
\hline Setor & Medianas & Demanda do Setor \\
\hline 1 & 6 & 6.491 \\
\hline 2 & 18 & 7.011 \\
\hline 3 & 29 & 7.329 \\
\hline 4 & 41 & 7.706 \\
\hline 5 & 51 & 6.628 \\
\hline 6 & 55 & 7.137 \\
\hline
\end{tabular}

Quadro 2: Comparação entre as demandas dos 6 Setores, abordagem 1, dezembro/04.

Os resultados obtidos mensalmente pelo método exato foram comparados com o método manual aplicado pela empresa e, em todas as situações, o modelo matemático mostrou-se mais adequado, isto é, as respostas apresentadas pelo algoritmo das $p$-medianas formavam Setores compostos somente por Rotas adjacentes (garantindo que a distância percorrida entre Rotas seja reduzida) e com menores diferenças entre as demandas máxima e mínima de solicitações (em minutos) por Setor, conforme pode ser observado no quadro 3.

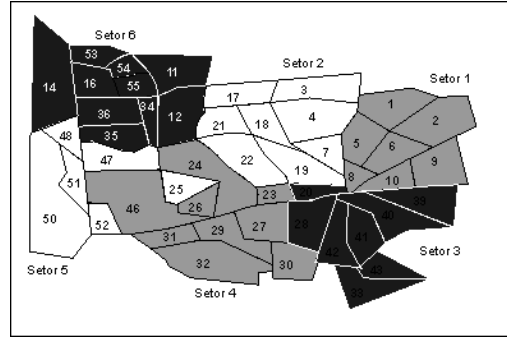

Figura 2: Divisão de Setores em dezembro, na abordagem 1, método exato.

\begin{tabular}{|c|c|c|c|c|c|c|}
\hline & Dez & Jan & Fev & Mar & Abr & Mai \\
\hline Situação atual & 5.343 & 5.448 & 5.439 & 5.200 & 5.726 & 6.111 \\
\hline Abordagem 1 & 1.215 & 1.992 & 1.049 & 1.716 & 2.750 & 1.227 \\
\hline Abordagem 2 & 1.288 & 1.733 & 1.606 & 3.913 & 3.499 & 1.559 \\
\hline
\end{tabular}

Quadro 3: Diferença, em minutos, entre as equipes com mais e menos solicitações.

Analisando-se os resultados ótimos do semestre, obtidos no modelo, observou-se grande semelhança entre os agrupamentos mensais sugeridos pelo algoritmo das $p$ medianas capacitado, o que motivou a busca de uma solução média para o problema, que representasse o semestre inteiro e pudesse ser utilizada futuramente na referida Agência.

A média semestral de solicitações por Rota foi calculada para servir de dado de entrada para novas simulações no método exato utilizado. E para confirmar a aplicabilidade do modelo à situações futuras, utilizou-se o número de solicitações 
do mês de junho no agrupamento obtido pela média de solicitações do semestre anterior. Como em junho de 2005 a agência passou a contar com apenas 5 equipes (figura 3a), as simulações utilizando a média foram feitas para o caso de 5 Setores.

Se a nova divisão da Agência em 5 Setores tivesse sido feita utilizando-se as informações do semestre anterior (média de solicitações por Rota) e os agrupamentos sugeridos pelo modelo matemático, (figura 3b), ter-se-ia uma melhor distribuição de solicitações por equipe isto é, a diferença entre os Setores mais e menos solicitados cairia de 4.780 para 2.992 minutos, o que equivale à uma redução de 29,8 horas ou seja, 3,73 dias entre os dois métodos.

O quadro 4 mostra as demandas de solicitações do mês de junho, por Setor, aplicadas ao método manual (figura 3a) e às duas abordagens matemáticas (figura $3 \mathrm{~b})$.

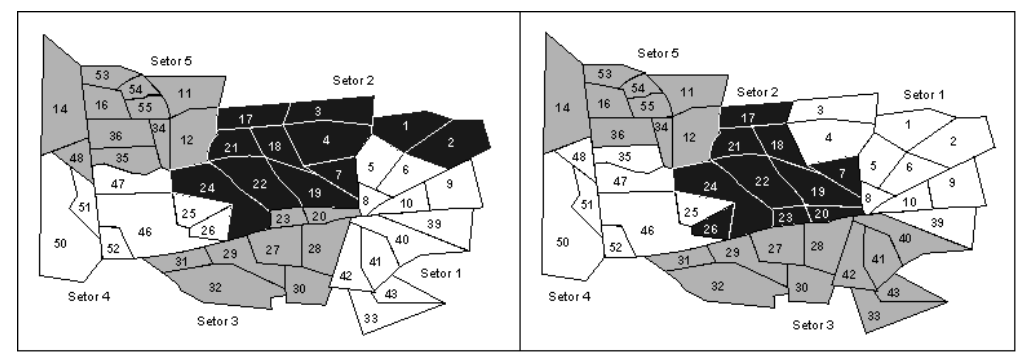

Figura 3 a) divisão manual da Agência em 5 Setores; b) melhor resposta, abordagem 2, utilizando-se a média semestral de solicitações.

\begin{tabular}{|c|c|c|c|c|c|c|}
\hline Setor & 1 & 2 & 3 & 4 & 5 & Maior diferença \\
\hline Situação atual & 11.862 & 9.854 & 7.082 & 10.681 & 11.901 & 4.780 \\
\hline Abordagem 1 & 11.625 & 8.471 & 10.810 & 8.123 & 12.351 & 4.228 \\
\hline Abordagem 2 & 11.768 & 8.776 & 9.827 & 11.004 & 10.005 & 2.992 \\
\hline
\end{tabular}

Quadro 4: Comparação entre situação atual e simulações, mês de junho, 5 Setores.

\subsection{Simulações do Método Heurístico}

As respostas obtidas através do algoritmo genético para 5 e 6 Setores de Atendimento, são mostradas nos quadros 5 e 6 , respectivamente.

\begin{tabular}{|c|c|c|c|c|c|c|}
\hline Mês & Dez & Jan & Fev & Mar & Abr & Mai \\
\hline Situação atual & 2.781 & 3.836 & 3.405 & 1.289 & 5.729 & 5.260 \\
\hline Algoritmo Genético & 1.244 & 1.704 & 2.895 & 3.445 & 1.596 & 1.940 \\
\hline
\end{tabular}

Quadro 5: Diferença (em minutos) entre as equipes com mais e menos solicitações, 5 Setores. 


\begin{tabular}{|c|c|c|c|c|c|c|}
\hline Mês & Dez & Jan & Fev & Mar & Abr & Mai \\
\hline Situação atual & 5.343 & 5.448 & 5.439 & 5.200 & 5.726 & 6.111 \\
\hline Algoritmo Genético & 758 & 1.940 & 4.039 & 3.053 & 1.529 & 1.698 \\
\hline
\end{tabular}

Quadro 6: Diferença (em minutos) entre as equipes com mais e menos solicitações, 6 Setores.

Observa-se, no quadro 5, que março foi o único mês do semestre para o qual o método manual atualmente utilizado pela Agência (5 Setores), apresentaria uma solução melhor do que o A.G. Para a situação com 6 Setores, todas as respostas obtidas através do A.G. apresentaram uma melhor divisão de tarefas por equipe, quando comparadas com o modelo manual utilizado até maio de 2005, conforme verifica-se no quadro 6.

Analogamente ao efetuado no modelo exato, utilizou-se a média de solicitações para gerar uma solução média para o problema. O melhor agrupamento médio obtido é mostrado no mapa da figura 4.

Com base nos dados do mês de junho de 2005, conclui-se que se a nova divisão da Agência em 5 Setores tivesse sido feita utilizando-se as informações do semestre anterior (média de solicitações por Rota) e o melhor agrupamento obtido pela heurística (figura 4), a diferença (em minutos) entre os Setores mais e menos solicitados cairia de 4.780 para 2.332 minutos (quadro 7), o que equivale à uma redução de 40,8 horas ou seja, 5,1 dias, entre os dois métodos.

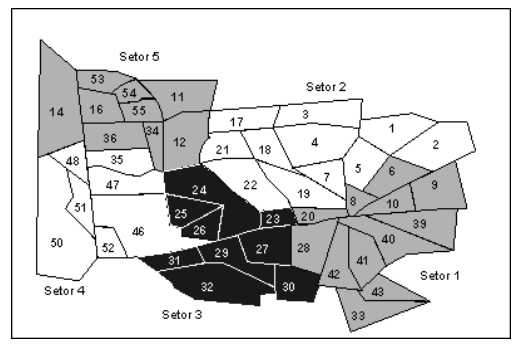

Figura 4: Melhor agrupamento médio sugerido pela heurística.

\begin{tabular}{|c|c|c|c|c|c|c|}
\hline Setor & 1 & 2 & 3 & 4 & 5 & Maior diferença \\
\hline Situação atual & 11.862 & 9.854 & 7.082 & 10.681 & 11.901 & 4.780 \\
\hline Heurística & 11.747 & 10.753 & 9.460 & 10.005 & 9.415 & 2.332 \\
\hline
\end{tabular}

Quadro 7: Comparação entre situação atual e simulações, mês de junho, 5 Setores.

\subsection{Comparação entre os métodos}

Analisando-se as soluções obtidas para o problema através dos métodos exato e heurístico, observa-se a viabilidade de ambos.

O quadro 8 mostra a diferença entre os Setores com maior e menor demanda de solicitações em cada método e os desvios padrão, aplicando-se os dados de junho de 2005 aos melhores agrupamentos obtidos. Observa-se no referido quadro que o 
Algoritmo Genético apresentou resposta melhor que o modelo matemático exato (resposta ótima), o que é explicado pelo fato do AG não buscar a menor distância percorrida entre os vértices do grafo, mas um agrupamento conexo de Rotas de Leitura adjacentes, para a formação de Setores de Atendimento.

A aplicação dos dados do mês de junho nos agrupamentos determinados manual e matematicamente, mostrou que as respostas dos modelos matemáticos foram mais adequadas que as do método manual utilizado pela Agência.

\begin{tabular}{|c|c|c|c|c|c|c|c|}
\hline Setor & 1 & 2 & 3 & 4 & 5 & $\begin{array}{c}\text { Maior } \\
\text { Diferença }\end{array}$ & $\begin{array}{c}\text { Desvio } \\
\text { Padrão }\end{array}$ \\
\hline $\begin{array}{c}\text { Método } \\
\text { Manual }\end{array}$ & 11.862 & 9.854 & 7.082 & 10.681 & 11.901 & 4.780 & 3.962 \\
\hline $\begin{array}{c}\text { Modelo } \\
\text { Exato }\end{array}$ & 11.768 & 8.776 & 9.827 & 11.004 & 10.005 & 2.992 & 2.298 \\
\hline Heurística & 11.747 & 10.753 & 9.460 & 10.005 & 9.415 & 2.332 & 1.968 \\
\hline
\end{tabular}

Quadro 8: Comparação entre maior e menor demanda de solicitações, dados de junho/05.

As respostas apresentadas pela heurística desenvolvida adequam-se bem ao problema, viabilizando sua utilização pela empresa, não justificando a necessidade de aquisição de um software específico para resolver o problema de maneira exata.

\section{Conclusões}

Os resultados obtidos neste trabalho mostraram-se satisfatórios tanto para o método exato quanto para o heurístico, pois os novos agrupamentos sugeridos mostraramse mais eficientes do que os vigentes, determinados manualmente e de maneira empírica.

O modelo exato das $p$-medianas tem como vantagem apresentar Setores com distâncias mínimas entre suas Rotas, o que é muito interessante, pois tal característica pode reverter em ganhos financeiros para a empresa que, ao ter seus veículos distribuídos de forma otimizada, economizaria em combustível e adiaria possíveis manutenções em sua frota. Porém, tem a desvantagem de requerer software específico, não disponível na empresa.

O método heurístico desenvolvido no presente trabalho também mostrou-se adequado ao problema, pois, em alguns casos, apresentou respostas semelhantes às do método exato. Salienta-se que o algoritmo implementado não é um algoritmo genético para solução das $p$-medianas, como é facilmente encontrado na literatura, mas um algoritmo adequado à formação de agrupamentos compactos, sem o inconveniente de formação de sub-setores desconexos num mesmo Setor de Atendimento. Tal metodologia não foi encontrada na literatura consultada para o desenvolvimento deste trabalho.

A característica probabilística dos dados utilizados (número de solicitações mensais por Rota de Leitura) dificulta previsões futuras, sugerindo que a utilização de um histórico maior de solicitações por Rota aumentaria a eficácia dos algoritmos 
para aplicação a situações futuras, possibilitando sua possível utilização nas demais agências da COPEL.

Abstract. This work proposes the use of techniques of operational research to determine clients groupings of an electric energy dealer. The objective is to reduce the time of execution of the services request by the clients through a better distribuition of tasks between the available team for such. The results were obtained through the use of p-median with capacity algorithm and a heuristic based on genetic algorithms. Several tests have shown that the applied mathematical models solve the problem better than the manual methods that are currently used by the company.

\section{Referências}

[1] E. Bobel, "Dimensionamento e Locação de Equipes de Manutenção em Redes de Distribuição de Energia Elétrica", Dissertação de Mestrado, CEFET, Curitiba, PR, 2003.

[2] L. Bodin, Golden, Bruce, A. Arjang, M. Ball, "Routing and Scheduling of Vehicles and Crews: the state of the art", Pergamon Press, England, 1983.

[3] N. Christofides, "Graph Theory: an algorithmic approach", Academic Press, New York, 1975.

[4] A.R.T. Goés, "Otimização na Distribuição da Carga Horária de Professores método exato, método heurístico, método misto e interface", Dissertação de Mestrado, UFPR, Curitiba, PR, 2005.

[5] B. Golden, T. Magnanti, H. Nbuyen, Implementing vehicle routing algorithms, Networks, 7 (1977).

[6] J.J. Grefenstette, Optimization of control parameters for genetic algorithms, IEE Transactions on Systems, Man and Cybernetics, 16, No. 1 (1986), 122-128.

[7] M. Hojati, Optimal Political Districting, Computers Operational Research, 23, No. 12 (1996), 1147- 1161.

[8] L.A.N. Lorena, E.L.F. Senne, J.A.C. Paiva, M.A. Pereira, Integração de Modelos de Localização a sistemas de Informações Geográficas, em Gestão e Produção, 8 (2001), 180-195. 2001.

[9] N.D. Pizzolato, F.B. Barcelos, L.A.N. Lorena, "Localização de Escolas do Ensino Fundamental com Modelos Capacitado e Não Capacitado: Caso de Vitória/ES", in: SOBRAPO, v.24, No. 1. Rio de Janeiro, RJ, 2004. 